\title{
A Liouville Type Result for Schrödinger Equation on Half-Spaces
}

\author{
Baiyu Liu \\ School of Mathematics and Physics, University of Science and Technology, Beijing, 30 Xueyuan Road, Haidian District, \\ Beijing 100083, China \\ Correspondence should be addressed to Baiyu Liu; liubymath@gmail.com
}

Received 10 September 2013; Accepted 20 November 2013

Academic Editor: Svatoslav Staněk

Copyright (C) 2013 Baiyu Liu. This is an open access article distributed under the Creative Commons Attribution License, which permits unrestricted use, distribution, and reproduction in any medium, provided the original work is properly cited.

We consider a nonlinear Schrödinger equation with a singular potential on half spaces. Using a Hardy-type inequality and the moving plane method, we obtain a Liouville type result for its nonnegative solutions.

\section{Introduction}

Recently, properties of nontrivial solutions for nonlinear elliptic equations on half spaces have attracted a great deal of attention from physicians and mathematicians; see, for example, [1-5].

In this paper, we consider nonnegative solutions of the following Schrödinger equation with a singular potential on the half-space:

$$
\begin{gathered}
-\Delta u-\frac{\beta}{z^{2}} u-u^{2^{*}-1}=0, \quad x \in H, \\
u=0, \quad x \in \partial H,
\end{gathered}
$$

where $n \geq 3,2^{*}=2 n /(n-2), \beta>0$, and

$$
H=\mathbb{R}_{+}^{n}=\left\{x=\left(x^{\prime}, z\right) \mid x^{\prime} \in \mathbb{R}^{n-1}, z>0\right\} .
$$

Equation (1) is related to the Grushin type equation with critical exponent and the Webster scalar curvature equation $[6,7]$.

We are interested in the Liouville type result for nonnegative solutions of (1). This work is motivated by some monotonicity results and Liouville type results for elliptic equations on half-spaces; see, for example, [2,3]. In [2], Dancer found some sufficient conditions for nonlinear term $f(u)$ such that the positive bounded solution $u$ of $-\Delta u=f(u)$ with Dirichlet boundary value condition is monotone increasing in $z$. Guo [3] considered nonnegative solutions for the elliptic system,

$$
\begin{aligned}
& -\Delta u=f(v), \quad \text { in } \mathbb{R}_{+}^{n}, \\
& -\Delta v=g(u), \quad \text { in } \mathbb{R}_{+}^{n}, \\
& u=v=0, \quad \text { on } \partial \mathbb{R}_{+}^{n},
\end{aligned}
$$

and obtained some sufficient conditions for $f$ and $g$, under which system (3) admits only trivial solution.

Let $\mathscr{D}_{0}^{1,2}(H)$ be the space given by the completion of $C_{0}^{\infty}(H)$ under the norm $\|u\|=\left(\int_{H}|\nabla u|^{2} d x\right)^{1 / 2}$. We say that $u$ is a weak solution of (1) if $u \in \mathscr{D}_{0}^{1,2}(H)$ satisfies

$$
\int_{H} \nabla u \cdot \nabla \varphi d x=\int_{H} \frac{\beta}{z^{2}} u \varphi d x+\int_{H} u^{2^{*}-1} \varphi d x,
$$

for all $\varphi \in C_{c}^{\infty}(H)$.

Using a Hardy-type inequality and the moving plane method in integral forms [8-10], we obtain the following Liouville type result.

Theorem 1. Let $u \in \mathscr{D}_{0}^{1,2}(H)$ be a nonnegative weak solution of (1) with $0<\beta<1 / 16$. Then, $u \equiv 0$.

Remark 2. For a weak solution $u \in \mathscr{D}_{0}^{1,2}(H)$, by using a regularity lifting method [8], we know that $u \in C^{2, \alpha}(\Omega)$, for all bounded smooth domain $\Omega \subset H$. Hence, it is a classical solution. 


\section{Preliminary}

In this section, we prepare some lemmas.

Firstly, we recall the Hardy-Sobolev inequality in the half space; see [11-13].

Lemma 3. Let $u \in \mathscr{D}_{0}^{1,2}\left(\mathbb{R}_{+}^{n}\right)$; then,

$$
\int_{\mathbb{R}_{+}^{n}} \frac{|u|^{2}}{z^{2}} d x \leq 4 \int_{\mathbb{R}_{+}^{n}}|\nabla u|^{2} d x .
$$

This inequality plays a crucial role in estimating the singular potential term in the following proof.

In the following, we assume that $u \in \mathscr{D}_{0}^{1,2}(H)$ is a nonnegative weak solution of (1) with $0<\beta<1 / 16$. We are going to use the method of moving plane in the half-space.

For each $\lambda>0$, let

$$
\Sigma_{\lambda}=\left\{\left(x^{\prime}, z\right) \mid x^{\prime} \in \mathbb{R}^{n-1}, z \in(0, \lambda)\right\}=\mathbb{R}^{n-1} \times(0, \lambda) .
$$

For $x \in \Sigma_{\lambda}$, we write $x^{\lambda}=\left(x_{1}, \ldots, x_{n-1}, 2 \lambda-z\right)$ which is the reflected point of $x$ with respect to the hyperplane $T_{\lambda}=\{x=$ $\left.\left(x^{\prime}, z\right) \mid z=\lambda\right\}$ and define

$$
u_{\lambda}(x)=u\left(x^{\lambda}\right), \quad w_{\lambda}(x)=u_{\lambda}(x)-u(x) .
$$

Then, direct computation gives

$$
\begin{aligned}
-\Delta w_{\lambda}(x)= & -\Delta u_{\lambda}(x)+\Delta u(x) \\
= & \frac{\beta}{(2 \lambda-z)^{2}} u_{\lambda}(x)+\left(u_{\lambda}(x)\right)^{2^{*}-1} \\
& -\frac{\beta}{z^{2}} u(x)-(u(x))^{2^{*}-1} \\
= & \frac{\beta}{(2 \lambda-z)^{2}} u_{\lambda}(x)-\frac{\beta}{z^{2}} u_{\lambda}(x) \\
& +\frac{\beta}{z^{2}} u_{\lambda}(x)-\frac{\beta}{z^{2}} u(x) \\
& +\left(u_{\lambda}(x)\right)^{2^{*}-1}-(u(x))^{2^{*}-1} \\
= & \frac{\beta}{z^{2}} w_{\lambda}(x)+\xi(x, \lambda) w_{\lambda}(x) \\
& +\beta\left(\frac{1}{(2 \lambda-z)^{2}}-\frac{1}{z^{2}}\right) u_{\lambda}(x) ;
\end{aligned}
$$

here $\xi(x, \lambda)=\left(\left(u_{\lambda}(x)\right)^{2^{*}-1}-u(x)^{2^{*}-1}\right) /\left(u_{\lambda}(x)-u(x)\right)$.

For $x \in \Sigma_{\lambda}$, we have $2 \lambda-z>z, 1 /(2 \lambda-z)^{2}<$ $1 / z^{2}, u_{\lambda}(x) \geq 0$, and $\beta>0$. Therefore,

$$
-\Delta w_{\lambda}(x) \leq \frac{\beta}{z^{2}} w_{\lambda}(x)+\xi(x, \lambda) w_{\lambda}(x) .
$$

Define $w_{\lambda}^{+}(x)=\max \left\{w_{\lambda}(x), 0\right\}$ and $w_{\lambda}^{-}(x)=$ $-\min \left\{w_{\lambda}(x), 0\right\}$. Clearly, $w_{\lambda}^{+}(x) \geq 0, w_{\lambda}^{-}(x) \geq 0$ and $w_{\lambda}(x)=w_{\lambda}^{+}(x)-w_{\lambda}^{-}(x)$. Define

$$
\Sigma_{\lambda}^{-}=\left\{x \in \Sigma_{\lambda} \mid w_{\lambda}(x)<0\right\} .
$$

The heart of our argument is the following lemma.
Lemma 4. There exists a $C_{0}>0$, such that, for $\lambda>0$, if $\left\|w_{\lambda}^{-}\right\|_{L^{2^{*}\left(\Sigma_{\lambda}\right)}}>0$, then

$$
\|u(x)\|_{L^{2^{*}}\left(\Sigma_{\lambda}^{-}\right)} \geq C_{0} .
$$

Proof. For $0<\epsilon<\lambda / 4$, let $\eta_{\epsilon}(z) \in C\left(\mathbb{R}^{+}\right)$be defined by

$$
\eta_{\epsilon}(z)= \begin{cases}1, & z>\epsilon, \\ \frac{\log z-2 \log \epsilon}{-\log \epsilon}, & \epsilon^{2} \leq z \leq \epsilon, \\ 0, & z<\epsilon^{2} .\end{cases}
$$

Testing (9) in $\Sigma_{\lambda}$ with function $\eta_{\epsilon}^{2} w_{\lambda}^{-}$, we obtain

$$
\begin{gathered}
-\int_{\Sigma_{\lambda}} \Delta w_{\lambda}(x) \eta_{\epsilon}^{2} w_{\lambda}^{-} d x \leq \beta \int_{\Sigma_{\lambda}} \frac{1}{z^{2}} w_{\lambda}(x) \eta_{\epsilon}^{2} w_{\lambda}^{-} d x \\
+\int_{\Sigma_{\lambda}} \xi(x, \lambda) w_{\lambda}(x) \eta_{\epsilon}^{2} w_{\lambda}^{-} d x .
\end{gathered}
$$

The left hand side of (13) is

$$
\begin{aligned}
& -\int_{\Sigma_{\lambda}} \Delta w_{\lambda}(x) \eta_{\epsilon}^{2} w_{\lambda}^{-} d x \\
& \quad=\int_{\Sigma_{\lambda}^{-}}\left|\nabla w_{\lambda}^{-}\right|^{2} \eta_{\epsilon}^{2} d x+2 \int_{\Sigma_{\lambda}^{-}} \eta_{\epsilon} w_{\lambda}^{-} \nabla w_{\lambda}(x) \cdot \nabla \eta_{\epsilon} d x .
\end{aligned}
$$

Hence, we derive

$$
\int_{\Sigma_{\lambda}^{-}}\left|\nabla w_{\lambda}^{-}\right|^{2} \eta_{\epsilon}^{2} d x \leq I+I I+I I I
$$

where

$$
\begin{aligned}
& I=\beta \int_{\Sigma_{\lambda}^{-}} \frac{1}{z^{2}} \eta_{\epsilon}^{2}\left(w_{\lambda}^{-}\right)^{2} d x, \\
& I I=\int_{\Sigma_{\lambda}^{-}} \xi(x, \lambda) \eta_{\epsilon}^{2}\left(w_{\lambda}^{-}\right)^{2} d x, \\
& I I I=-2 \int_{\Sigma_{\lambda}^{-}} \eta_{\epsilon} w_{\lambda}^{-} \nabla w_{\lambda}(x) \cdot \nabla \eta_{\epsilon} d x .
\end{aligned}
$$

Using Lemma 3, we have

$$
\begin{aligned}
I & =\beta \int_{\Sigma_{\lambda}^{-}} \frac{1}{z^{2}} \eta_{\epsilon}^{2}\left(w_{\lambda}^{-}\right)^{2} d x \\
& \leq 4 \beta \int_{\Sigma_{\lambda}^{-}}\left|\nabla\left(w_{\lambda}^{-} \eta_{\epsilon}\right)\right|^{2} d x \\
& \leq 8 \beta \int_{\Sigma_{\lambda}^{-}}\left(\eta_{\epsilon}^{2}\left|\nabla w_{\lambda}^{-}\right|^{2}+\left(w_{\lambda}^{-}\right)^{2}\left|\nabla \eta_{\epsilon}\right|^{2}\right) d x
\end{aligned}
$$

For $x \in \Sigma_{\lambda}^{-}, 0 \leq u_{\lambda}(x)<u(x), 0<\xi(x, \lambda)<((n+2) /(n-$ 2) $(u(x))^{4 /(n-2)}$, which implies

$$
\begin{aligned}
I I & =\int_{\Sigma_{\lambda}^{-}} \xi(x, \lambda) \eta_{\epsilon}^{2}\left(w_{\lambda}^{-}\right)^{2} d x \\
& \leq \frac{n+2}{n-2} \int_{\Sigma_{\lambda}^{-}}(u(x))^{4 /(n-2)} \eta_{\epsilon}^{2}\left(w_{\lambda}^{-}\right)^{2} d x .
\end{aligned}
$$


By using Hölder inequality, we verify that

$$
\begin{aligned}
I I \leq & \frac{n+2}{n-2}\left(\int_{\Sigma_{\lambda}^{-}} u^{2^{*}} \eta_{\epsilon}^{n / 2} d x\right)^{2 / n} \\
& \cdot\left(\int_{\Sigma_{\lambda}^{-}}\left(w_{\lambda}^{-}\right)^{2^{*}} \eta_{\epsilon}^{n /(n-2)} d x\right)^{(n-2) / n} \\
\leq & \frac{n+2}{n-2}\|u\|_{L^{2^{*}}\left(\Sigma_{\lambda}^{-}\right)}^{4 /(n-2)}\left\|w_{\lambda}^{-}\right\|_{L^{2^{*}}\left(\Sigma_{\lambda}^{-}\right)}^{2} \\
I I I= & -2 \int_{\Sigma_{\lambda}^{-}} \eta_{\epsilon} w_{\lambda}^{-} \nabla w_{\lambda} \cdot \nabla \eta_{\epsilon} d x \\
\leq & 2 \int_{\Sigma_{\lambda}^{-}} \eta_{\epsilon} w_{\lambda}^{-}\left|\nabla w_{\lambda}^{-}\right|\left|\nabla \eta_{\epsilon}\right| d x \\
\leq & \frac{1}{4} \int_{\Sigma_{\lambda}^{-}} \eta_{\epsilon}^{2}\left|\nabla w_{\lambda}^{-}\right|^{2} d x+4 \int_{\Sigma_{\lambda}^{-}}\left|\nabla \eta_{\epsilon}\right|^{2}\left(w_{\lambda}^{-}\right)^{2} d x .
\end{aligned}
$$

Putting (17), (19), and (20) into (15) and using the assumption $1<\beta<1 / 16$, we then deduce that

$$
\begin{gathered}
\int_{\Sigma_{\lambda}^{-}}\left|\nabla w_{\lambda}^{-}\right|^{2} \eta_{\epsilon}^{2} d x \leq 9 \int_{\Sigma_{\lambda}^{-}}\left|\nabla \eta_{\epsilon}\right|^{2}\left(w_{\lambda}^{-}\right)^{2} d x \\
\quad+4 \cdot \frac{n+2}{n-2}\|u\|_{L^{2^{*}}\left(\Sigma_{\lambda}^{-}\right)}^{4 /(n-2)}\left\|w_{\lambda}^{-}\right\|_{L^{2^{*}}\left(\Sigma_{\lambda}^{-}\right)}^{2}
\end{gathered}
$$

Moreover, by the Sobolev inequality, we know that

$$
\begin{aligned}
\left\|w_{\lambda}^{-} \eta_{\epsilon}\right\|_{L^{2^{*}}\left(\Sigma_{\lambda}^{-}\right)}^{2} & \leq C^{2}\left\|\nabla\left(w_{\lambda}^{-} \eta_{\epsilon}\right)\right\|_{L^{2}\left(\Sigma_{\lambda}^{-}\right)} \\
& \leq C^{2} \int_{\Sigma_{\lambda}^{-}}\left|\eta_{\epsilon} \nabla w_{\lambda}^{-}+w_{\lambda}^{-} \nabla \eta_{\epsilon}\right|^{2} d x \\
& \leq 2 C^{2} \int_{\Sigma_{\lambda}^{-}}\left(\eta_{\epsilon}^{2}\left|\nabla w_{\lambda}^{-}\right|^{2}+\left(w_{\lambda}^{-}\right)^{2}\left|\nabla \eta_{\epsilon}\right|^{2}\right) d x
\end{aligned}
$$

Combine the above inequality with (21) to get

$$
\begin{aligned}
\left\|w_{\lambda}^{-} \eta_{\epsilon}\right\|_{L^{2^{*}}\left(\Sigma_{\lambda}^{-}\right)}^{2} \leq & 20 C^{2} \int_{\Sigma_{\lambda}^{-}}\left|\nabla \eta_{\epsilon}\right|^{2}\left(w_{\lambda}^{-}\right)^{2} d x \\
& +8 C^{2} \frac{n+2}{n-2}\|u\|_{L^{2^{*}}\left(\Sigma_{\lambda}^{-}\right)}^{(n-2) / 4}\left\|w_{\lambda}^{-}\right\|_{L^{2^{*}}\left(\Sigma_{\lambda}^{-}\right)}^{2} .
\end{aligned}
$$

Now we claim that

$$
\int_{\Sigma_{\lambda}^{-}}\left|\nabla \eta_{\epsilon}\right|^{2}\left(w_{\lambda}^{-}\right)^{2} d x \longrightarrow 0, \quad \text { as } \epsilon \longrightarrow 0
$$

Notice that, for $x \in \Sigma_{\lambda}^{-}, 0<w_{\lambda}^{-}(x)=u(x)-u_{\lambda}(x) \leq u(x)$. Hence,

$$
\begin{aligned}
0 & \leq \int_{\Sigma_{\lambda}^{-}}\left|\nabla \eta_{\epsilon}\right|^{2}\left(w_{\lambda}^{-}\right)^{2} d x \\
& \leq \int_{\Sigma_{\lambda}^{-}}\left|\eta_{\epsilon}^{\prime}(z)\right|^{2} u^{2} d x \\
& =\int_{\epsilon^{2} \leq z \leq \epsilon} \frac{(u(x))^{2}}{z^{2}(\log \epsilon)^{2}} d x \\
& \leq \frac{1}{(\log \epsilon)^{2}} \int_{H} \frac{(u(x))^{2}}{z^{2}} d x \\
& \leq 4 \frac{1}{(\log \epsilon)^{2}} \int_{H}|\nabla u|^{2} d x .
\end{aligned}
$$

Since $u \in \mathscr{D}_{0}^{1,2}(H), \int_{H}|\nabla u|^{2} d x<+\infty$. Thus (24) is valid.

Now, letting $\epsilon \rightarrow 0$ in (23), by using dominated convergence theorem, we obtain

$$
1 \leq 8 C^{2} \cdot \frac{n+2}{n-2}\|u\|_{L^{2^{*}}\left(\Sigma_{\lambda}^{-}\right)}^{4 /(-2)}
$$

if $\left\|w_{\lambda}^{-}\right\|_{L^{2^{*}\left(\Sigma_{\lambda}^{-}\right)}} \neq 0$.

One can choose $C_{0}=\left((n-2) / 8 C^{2}(n+2)\right)^{(n-2) / 4}$, where $C$ is the best constant in the Sobolev inequality.

Using Lemma 4, we now can start the moving plane process as the following Lemma.

Lemma 5. There is a $\lambda_{0}>0$, such that, for all $0<\lambda<\lambda_{0}$,

$$
w_{\lambda}(x) \geq 0, \quad \forall x \in \Sigma_{\lambda} .
$$

Proof. Since $u \in \mathscr{D}_{0}^{1,2}(H)$, using Sobolev inequality, we have $u(x) \in L^{2^{*}}(H)$. Choose $\lambda_{0}>0$ small enough such that

$$
\|u\|_{L^{2^{*}}\left(\Sigma_{\lambda_{0}}\right)}<C_{0}
$$

where $C_{0}$ is the same as in Lemma 4 .

Hence, for all $0<\lambda<\lambda_{0}$,

$$
\|u\|_{L^{2^{*}}\left(\Sigma_{\lambda}^{-}\right)} \leq\|u\|_{L^{2^{*}\left(\Sigma_{\lambda}\right)}} \leq\|u\|_{L^{2^{*}\left(\Sigma_{\lambda_{0}}\right)}}<C_{0}
$$

which is a contradiction to Lemma 4 , if $\left\|w_{\lambda}^{-}\right\|_{L^{2^{*}\left(\Sigma_{\lambda}^{-}\right)}} \neq 0$. That is to say,

$$
\left\|w_{\lambda}^{-}\right\|_{L^{2^{*}\left(\Sigma_{\lambda}^{-}\right)}}=0
$$

which implies that $w_{\lambda} \geq 0$, for $x \in \Sigma_{\lambda}$.

Now we move the hyperplane $T_{\lambda}$ upwards by increasing the value of $\lambda$ continuously as long as (27) holds. We will show that the hyperplane will be moved to the infinity. Precisely, define

$$
\Lambda=\sup \left\{\lambda>0 \mid w_{\mu}(x) \geq 0, \forall x \in \Sigma_{\mu}, \forall 0<\mu \leq \lambda\right\} .
$$

By the result of Lemma $5, \Lambda \geq \lambda_{0}>0$. 
Lemma 6. We have $\Lambda=+\infty$.

Proof. Suppose $\Lambda<+\infty$.

On one hand, by continuity we know that $w_{\Lambda}(x) \geq 0$, for all $x \in \Sigma_{\Lambda}$, which means

$$
\Sigma_{\Lambda}^{-}=\emptyset
$$

On the other hand, by the definition of $\Lambda$, there is $\left\{\delta_{i}\right\}_{i=1}^{\infty}$ that satisfy (i) $\delta_{i} \rightarrow 0$, as $i \quad \rightarrow \infty$, and (ii) $\left\|w_{\Lambda+\delta_{i}}^{-}(x)\right\|_{L^{2^{*}}\left(\Sigma_{\Lambda+\delta_{i}}\right)}>0$, for all $i$. By Lemma 4 , we get $\|u(x)\|_{L^{2^{*}}\left(\Sigma_{\Lambda+\delta_{i}}^{-}\right)} \geq C_{0}>0$. By using the dominated convergence theorem, we obtain

$$
\|u(x)\|_{L^{2^{*}}\left(\Sigma_{\Lambda}^{-}\right)} \geq C_{0}>0,
$$

which is a contradiction to (32).

\section{Proof of Theorem 1}

In this section, we prove Theorem 1.

Since $u$ is a superharmonic continuous function in $H$ (see Remark 2), we have either $u \equiv 0$ in $H$ or $u>$ 0 in $H$.

If $u>0$ in $H$, then there is some $\left(x_{0}^{\prime}, z_{0}\right) \in H$ satisfying $u\left(x_{0}^{\prime}, z_{0}\right)=c>0$. Moreover, by continuity, there is a $\delta>0$, such that $u\left(x^{\prime}, z_{0}\right)>a / 2$, for all $\left|x^{\prime}-x_{0}^{\prime}\right|<\delta$. By using Lemma 6 , we know that $u(x)$ is increasing with respect to $z$ in $H$. Thus, $u\left(x^{\prime}, z\right) \geq a / 2$ for all $\left|x^{\prime}-x_{0}^{\prime}\right|<\delta$ and $z \geq$ $z_{0}$. Hence,

$$
\begin{aligned}
& \int_{z_{0}}^{+\infty} \int_{\left|x^{\prime}-x_{0}^{\prime}\right|<\delta}\left|u\left(x^{\prime}, z\right)\right|^{2^{*}} d x^{\prime} d z \\
& \quad \geq \int_{z_{0}}^{+\infty} \int_{\left|x^{\prime}-x_{0}^{\prime}\right|<\delta}\left(\frac{a}{2}\right)^{2^{*}} d x^{\prime} d z=+\infty
\end{aligned}
$$

which contradicts the fact that $u \in L^{2^{*}}(H)$.

Therefore, $u \equiv 0$ in $H$.

\section{Acknowledgments}

This project is supported by the National Natural Science Foundation of China (no. 11201025) and the Fundamental Research Funds for the Central Universities (FRF-TP-12106A).

\section{References}

[1] L. Caffarelli and L. Silvestre, "An extension problem related to the fractional Laplacian," Communications in Partial Differential Equations, vol. 32, no. 7-9, pp. 1245-1260, 2007.

[2] E. N. Dancer, "Some notes on the method of moving planes," Bulletin of the Australian Mathematical Society, vol. 46, no. 3, pp. 425-434, 1992.

[3] Y. Guo, "Non-existence, monotonicity for positive solutions of semilinear elliptic system in $\mathbb{R}_{+}^{N}$," Communications in Contemporary Mathematics, vol. 12, no. 3, pp. 351-372, 2010.
[4] B. Liu and L. Ma, "Symmetry results for elliptic Schrödinger systems on half spaces," Journal of Mathematical Analysis and Applications, vol. 401, no. 1, pp. 259-268, 2013.

[5] B. Liu and L. Ma, "Symmetry results for decay solutions of semilinear elliptic systems on half spaces," Nonlinear Analysis: Theory, Methods \& Applications, vol. 75, no. 6, pp. 3167-3177, 2012.

[6] D. Castorina, I. Fabbri, G. Mancini, and K. Sandeep, "HardySobolev extremals, hyperbolic symmetry and scalar curvature equations," Journal of Differential Equations, vol. 246, no. 3, pp. 1187-1206, 2009.

[7] S. Chen and L. Lin, "Results on entire solutions for a degenerate critical elliptic equation with anisotropic coefficients," Science China, vol. 54, no. 2, pp. 221-242, 2011.

[8] W. Chen and C. Li, Methods on Nonlinear Elliptic Equations, vol. 4, American Institute of Mathematical Sciences (AIMS), 2010.

[9] Y. Guo and J. Liu, "Liouville type theorems for positive solutions of elliptic system in $\mathbb{R}^{N}$," Communications in Partial Differential Equations, vol. 33, no. 1-3, pp. 263-284, 2008.

[10] C. Li and L. Ma, "Uniqueness of positive bound states to Schrödinger systems with critical exponents," SIAM Journal on Mathematical Analysis, vol. 40, no. 3, pp. 1049-1057, 2008.

[11] S. Chen and S. Li, "Hardy-Sobolev inequalities in half-space and some semilinear elliptic equations with singular coefficients," Nonlinear Analysis: Theory, Methods \& Applications, vol. 66, no. 2, pp. 324-348, 2007.

[12] J. Tidblom, "A Hardy inequality in the half-space," Journal of Functional Analysis, vol. 221, no. 2, pp. 482-495, 2005.

[13] V. G. Maz’ja, Sobolev Spaces, Springer, Berlin, germany, 1985. 


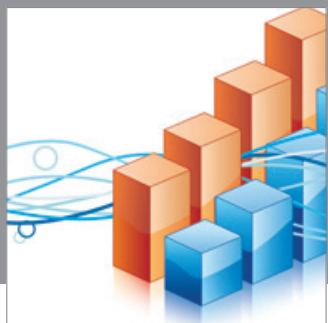

Advances in

Operations Research

mansans

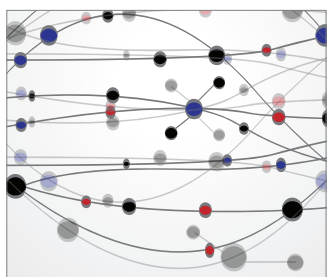

The Scientific World Journal
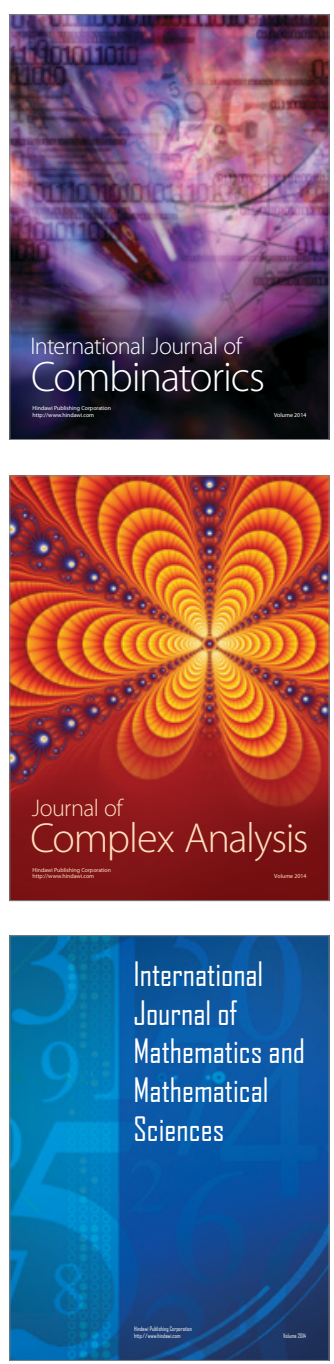
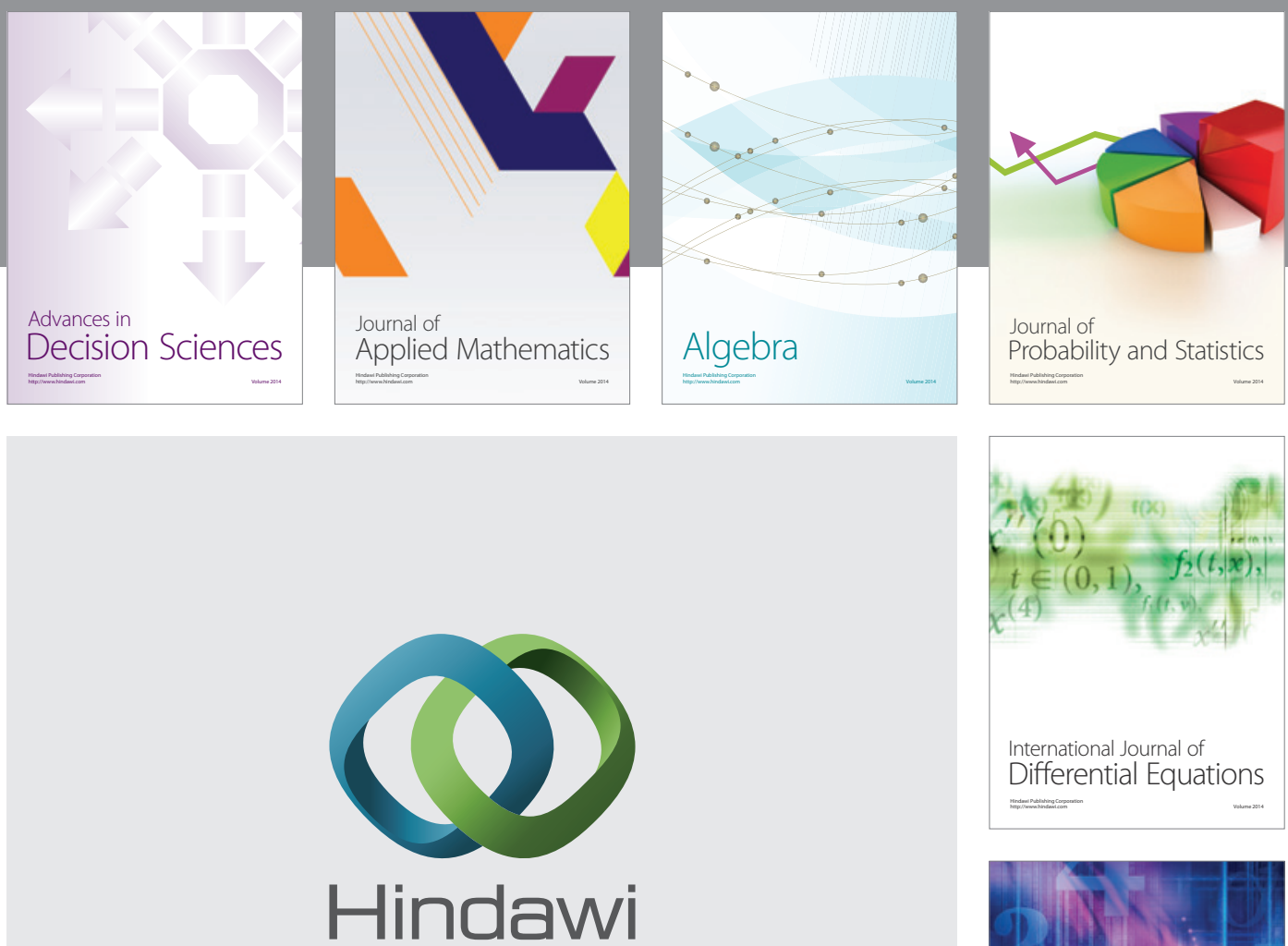

Submit your manuscripts at http://www.hindawi.com
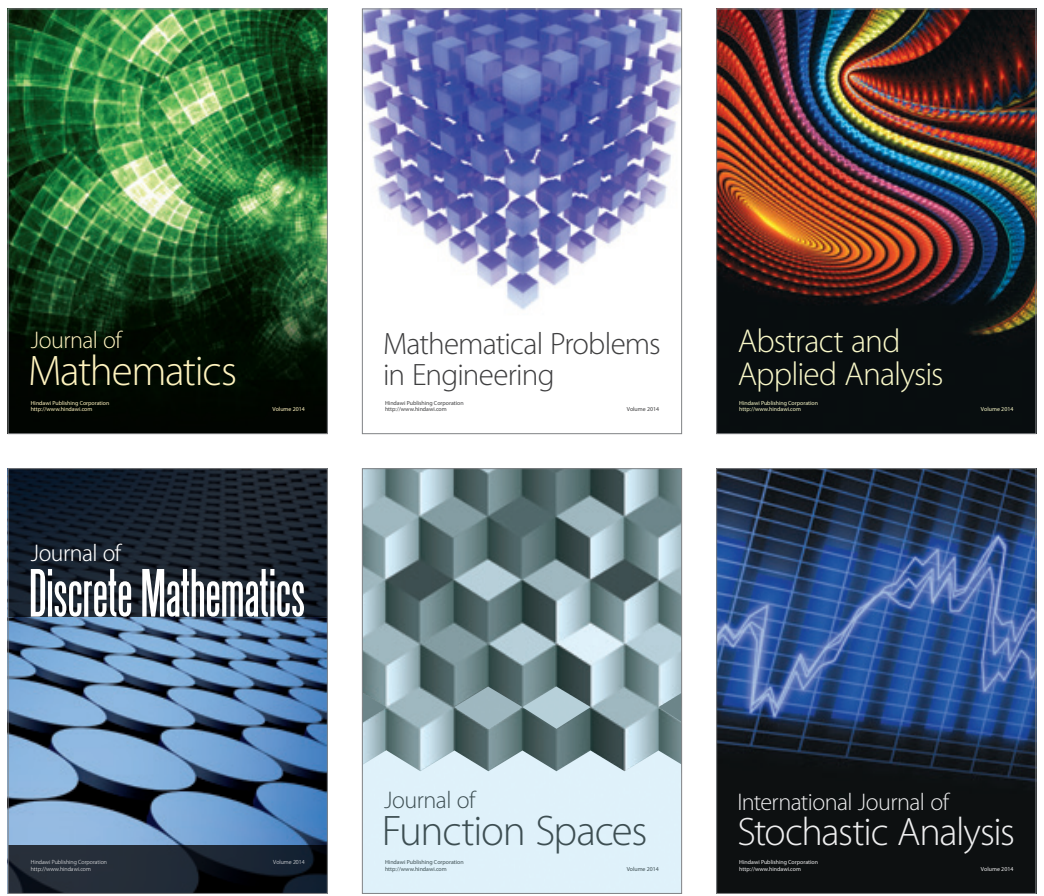

Journal of

Function Spaces

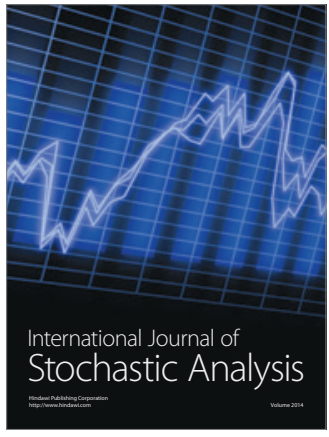

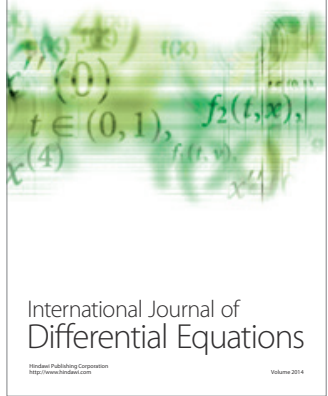
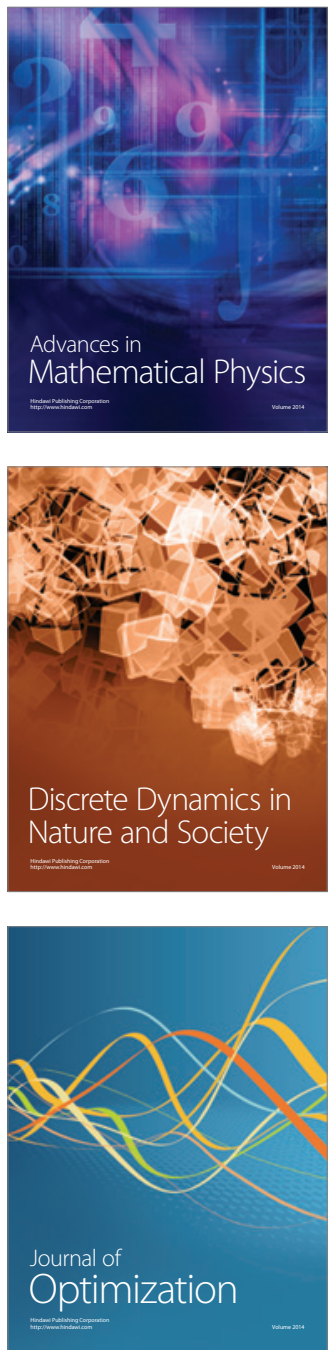